Hydrol. Earth Syst. Sci., 13, 1519-1530, 2009

www.hydrol-earth-syst-sci.net/13/1519/2009/

(c) Author(s) 2009. This work is distributed under

the Creative Commons Attribution 3.0 License.

\title{
Observed drought and wetness trends in Europe: an update
}

\author{
I. Bordi ${ }^{1}$, K. Fraedrich ${ }^{2, *}$, and A. Sutera ${ }^{1}$ \\ ${ }^{1}$ Department of Physics, University of Rome "La Sapienza", Italy \\ ${ }^{2}$ Meteorologisches Institut, KlimaCampus, Universität Hamburg, Hamburg, Germany \\ *also at: Max Planck Institute for Meteorology, Hamburg, Germany
}

Received: 8 May 2009 - Published in Hydrol. Earth Syst. Sci. Discuss.: 20 May 2009

Revised: 8 August 2009 - Accepted: 17 August 2009 - Published: 25 August 2009

\begin{abstract}
Linear and nonlinear trends of drought and wetness are analysed in terms of the gridded Standardized Precipitation Index (SPI) determined from monthly precipitation in Europe (NCEP/NCAR). In characterizing the meteorological and hydrological aspects, the index is computed on a seasonal and on a bi-annual time scale. Two datasets are compared: one from 1949 to 1997 and the other one includes the update of the last decade (to February 2009). The following results are noted: (i) time series of drought and wetness area coverage (number of grid points above/below the severity threshold) show a remarkable linear trend until about the end of the last century, which is reversed in the last (update) decade. This recent trend reversal is an indication of a nonlinear trend, which is more pronounced on the hydrological time scale. (ii) A nonlinear trend analysis is performed based on the time series of the principal component (PC) associated to the first spatial SPI-eigenvector after embedding it in a time delay coordinate system using a sliding window of 70 months (singular spectrum analysis). Nonlinearity appears as a clear feature on the hydrological time scale. (iii) The first spatial EOF-patterns of the shorter and the longer (updated) SPI time series fields show similar structure. An inspection of the SPI time behaviour at selected grid points illustrates the spatial variability of the detected trends.
\end{abstract}

\section{Introduction}

Drought is a natural and recurrent feature of climate that originates from a deficiency of precipitation over an extended period of time, usually a season or more. It should be considered relative to some long-term average conditions

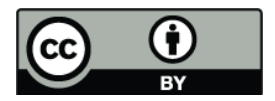

Correspondence to: I. Bordi (isabella.bordi@roma1.infn.it) often perceived as "normal". Thus, a dry spell, i.e. a period characterized by an abnormally dry weather lasting from several days to a few months, must be expected as part of the natural sequence of events. However, if a drier than usual period continues for many months or years it is unlikely this is part of the normal continuum of events, and we refer to it as a drought event (Pereira et al., 2009).

Usually, we tend to focus on drought when it is occurring and to react when crises strike. On the contrary, we should take a proactive approach to dealing with drought, anticipating the occurrence of the natural phenomenon and planning measures for minimizing its negative effects (Wilhite et al., 2000). However, making the transition from crisis to risk management is difficult because little has been done to understand and address the risks associated with drought, and to identify the factors that influence the vulnerability of a region to dry spells (Wilhite, 2002).

The degree of a region's vulnerability depends on many environmental and social factors as well as on the ability to anticipate, cope with and recover from drought. Among the environmental factors there is the natural climatic variability of the area, also in relation to climate change. Although we do not know how climate change will affect regional water resources, a first issue to shed light on this problem is the analysis of climatic trends evaluated using updated data. In water resources management, in fact, this is a crucial aspect for planning proactive mitigation measures against future drought occurrences (Hayes et al., 2004). Nevertheless, due to the shortness of the time records and problems related to the homogeneity of data sets, it is difficult to objectively estimate trends and their statistical significance, as well as to discern between linear trend and long-term periodicity.

As precipitation is an important water resources supply component, an analysis of precipitation characteristics is a critical component of drought risk. However, if we wish to compare climatic conditions of different areas, which

Published by Copernicus Publications on behalf of the European Geosciences Union. 
often are characterized by different hydrological balances, we need a standardized variable able to objectively capture the drought condition of a region. For this purpose the Standardized Precipitation Index (SPI) appears to be the most powerful drought index. It is based only on the precipitation field, it is standardized and can be computed on different time scales, allowing to monitor the various kinds of droughts (Keyantash and Dracup, 2002; Bordi and Sutera, 2007). Meteorological drought is usually an expression of precipitation departure from normal conditions over a period of time of a few months, while hydrological drought refers to deficiencies in surface and subsurface water supplies due to precipitation reduction over an extended period of time of one year or more. Thus, the different time scales used for the computation of the SPI reflect the impact of drought on the available water resources; typically 3 -month time scale is used to characterize meteorological conditions while 12 or 24-month time scales are used to monitor hydrological drought. Moreover, since the index is standardized wet conditions can be monitored as well.

Recently, a downward linear trend over most of Europe and Mediterranean basin has been unveiled in the SPI time series computed using the reanalysis data sets, implying an overall tendency towards drier periods from the seventies onward (Bordi and Sutera, 2001, 2002, 2004; Bordi et al., 2006). This general behaviour has been documented also in several studies carried out at regional level and based on gridded rain-gauge data or observations (see for example Szinell et al., 1998; Lloyd-Hughes and Saunders, 2002; VicenteSerrano and Cuadrat-Prats, 2007 and references therein). Furthermore, in the latest years most of European countries experienced several wet events, in contrast with the aforementioned tendency. Motivated by these evidences we present an analysis in Europe of the variability of the linear trend unveiled in the SPI time series updated to February 2009. After a preliminary investigation on meterorological drought using the SPI on 3-month time scale, the paper focuses on hydrological drought analyzed through the SPI on 24-month time scale. This is both for continuity with previous papers (Bordi and Sutera, 2001, 2004) and, because the long time scale filters out the effects on drought of short-term periodicities and seasonal cycle, thus enhancing the long-term variability, which is the goal of our study. For this purpose, we use the National Centers for Environmental Prediction/National Center for Atmospheric Research (NCEP/NCAR) reanalysis data that meet the fundamental requirements for an analysis of drought at large-scale. The data set, in fact, is freely accessible through the web, covers the globe uniformly, has a time duration sufficiently long to be trustworthy in a statistical sense, and it is continually updated. Furthermore, we apply the Singular Spectrum Analysis (SSA) to the time series of interest (that is, area coverage of dry/wet events, first principal component score of the SPI field and SPI time series at selected grid points) to extract the leading nonlinear components.
In the following sections there are a brief description of the data and methodology (Sect. 2), an illustration of the main results (Sects. 3 and 4) and a discussion with an outlook on future works (Sect. 5).

\section{Data and methods}

Data used for the analysis are monthly mean precipitation rates retrieved from the NCEP/NCAR reanalysis archive for the period January 1948-February 2009. They are available on a regular grid $1.9^{\circ} \times 1.9^{\circ}$ in longitude and latitude. Such precipitation data have been derived from the primary meteorological fields of the NCEP medium range forecasting spectral model with 28 "sigma" vertical levels and a triangular truncation of 62 waves, equivalent to about $210-\mathrm{km}$ horizontal resolution. The model is based on the assimilation of a set of observations, such as land surface, ship, rawinsonde, aircraft and satellite data (Kalnay et al., 1996). These data were quality controlled and assimilated with a data assimilation system kept unchanged over the reanalysis period. Though precipitation is not directly assimilated, but derived completely from the model 6-hour forecast, its midlatitude features have been compared favourably with observations and several climatologies (Janowiak et al., 1998; Trenberth and Guillemot, 1998). Since for the present study we have considered the area centred over Europe $\left(25.72^{\circ} \mathrm{N}-71.43^{\circ} \mathrm{N}\right.$, $13.13^{\circ} \mathrm{W}-60.00^{\circ} \mathrm{E}$ ), we may feel enough confidence on the data quality. However, it is worth to notice that although the reanalysis system remained essentially unchanged during the more than 60 years processed (apart minor corrections provided after some errors' detection), there were two major changes in the observing system. The first took place during 1948-1957, when the upper-air network was established, and the second in 1979 when the global operational use of satellite soundings was introduced (Kistler et al., 2001).

Meteorological dry/wet conditions over Europe, updated to February 2009, have been assessed through the SPI on 3month time scale, while 24-month time scale is used to investigate hydrological conditions. The SPI was introduced by McKee et al. (1993) to quantify the precipitation deficit for multiple time scales that reflect the impact of drought on the availability of the different water resources (Keyantash and Dracup, 2002; Heim, 2002). It is based on the precipitation field alone and its computation for any location is based on the long-term precipitation record cumulated over the selected time scale. This long-term record is fitted to a probability distribution (usually a Gamma distribution, Guttman, 1999), which is then transformed through an equalprobability transformation into a normal distribution. Positive SPI values indicate greater than median precipitation, and negative values indicate less than median precipitation (Bordi and Sutera, 2001). Thus, the SPI seems to be a useful tool for monitoring drought and wetness on multiple time 
scales and comparing climatic conditions of areas governed by different hydrological regimes.

In analyzing the long-term drought variability, we evaluate both the linear trends and the leading nonlinear components in the SPI time series. To extract the long-term linear trend we use the least-squared method to fit a linear model to the time series. Thus, at each grid point we have:

$\hat{y}=p_{1} t+p_{2}$

with $\hat{y}$ the fitted response, $p_{1}, p_{2}$ the angular coefficient and intercept of the linear model, and $t$ the time in years. The $R^{2}$ statistics, which measures how successful the fit is in explaining the variation of the data, is computed as:

$R^{2}=1-\frac{\mathrm{SSE}}{\mathrm{SST}}$

with SSE the sum of squared errors and SST the sum of squares about the mean.

The leading nonlinear components in the SPI time series are extracted using the Singular Spectral Analysis (SSA). This method better captures the intrinsic nonlinear behaviour of non-periodic and non-stationary signals with respect to other methods based on preselected basis functions, such as the Fourier transform or polynomial fitting. Moreover, since it is based on the principal component decomposition, the computed trend explains more variance than other techniques.

SSA, is a nonparametric spectral estimation method based on embedding a time series $X(t): t=1, N$ in a vector space of dimension $M$. It consists in diagonalizing the $M \times M$ lagcovariance matrix $\mathbf{C}_{\mathbf{X}}$ of $X(t)$ to obtain spectral information on the time series. The matrix $\mathbf{C}_{\mathbf{X}}$ can be estimated directly from the data as a Toeplitz matrix with constant diagonals (Vautard and Ghil, 1989), i.e. its entries $c_{i j}$ depend only on the lag $|i-j|$ :

$c_{i j}=\frac{1}{N-|i-j|} \sum_{t=1}^{N-|i-j|} X(t) X(t+|i-j|)$.

An alternative way to compute $\mathbf{C}_{\mathbf{X}}$ (Broomhead and King, 1986; Fraedrich, 1986) is by using the $N^{\prime} \times M$ "trajectory matrix" $\mathbf{D}$ that is formed by $M$ lag-shifted copies of $X(t)$, which are $N^{\prime}=N-M+1$ long; then

$\mathbf{C}_{\mathbf{X}}=\frac{1}{N^{\prime}} D^{T} \mathbf{D}$.

The $M$ eigenvectors $\boldsymbol{E}_{\boldsymbol{k}}$ of the lag-covariance $\mathbf{C}_{\mathbf{X}}$ are called temporal empirical orthogonal functions (EOFs), while the eigenvalues $\lambda_{k}$ of $\mathbf{C}_{\mathbf{X}}$ account for the partial variance in the direction of $\boldsymbol{E}_{\boldsymbol{k}}$. Projecting the time series onto each eigenvector yields the corresponding temporal principal component (PCs) $A_{k}$ :

$A_{k}(t)=\sum_{j=1}^{M} X(t+j-1) \boldsymbol{E}_{\boldsymbol{k}}(j)$
The entire time series or part of it that correspond to trends, oscillatory modes or noise can be reconstructed by using linear combinations of PCs and EOFs, as:

$R_{k}(t)=\frac{1}{M_{t}} \sum_{k \in K} \sum_{j=L_{t}}^{U_{t}} A_{k}(t-j+1) \boldsymbol{E}_{\boldsymbol{k}}(j)$

where $K$ is the set of EOFs on which the reconstruction is based. Values of the normalization factor $M_{t}$, as well as the lower and upper bound of summation $L_{t}$ and $U_{t}$, differ between the central part of the time series and its end points (Ghil et al., 2002).

In our case we generate the "trajectory matrix" from the time series of interest and reconstruct the signal considering only the leading component. For practical purposes we select a window length (of $M=70$ months), which is about $1 / 10$ th of the time series, because it provides statistically meaningful estimates (that is ten samples) of the largest resolvable fluctuation period. Time series of the Standardized Precipitation Index of 3 and 24 months, SPI-3 and SPI-24, are used for analysis characterizing drought and wetness aiming at the meteorological and hydrological applications.

Note that the conventional Principal Component Analysis (PCA or EOF analysis, Rencher, 1998) is a special case with $M=1$. In the present context we will apply the PCA to the SPI-24 field to extract the primary features of the space-time variability of drought and wetness (as for example in Bordi and Sutera, 2004). Note that the spatial EOF patterns, properly normalized, are called "loadings" and represent the correlations between PC scores and the observations (SPI time series in our case).

\section{Spatial pattern and time variability of linear and nonlinear trend}

Drought and wetness in the European region (Eurasia) are characterized by their spatial extent and evolution in time which, after combination provide the complete picture. Thus, at any prescribed time (month) both agricultural and hydrological drought and wetness in Europe are graded (quantified) in terms of (i) their fractional areas with SPI-values above and below their respective thresholds and (ii) by the principal component of the first (dominating) EOF of the European SPI-field. The subsequent trend analysis of these time series is based on the classical linear (regression) and nonlinear (SSA) techniques, comparing the previous trend with the recent update. First, the meteorologically relevant SPI3 features are analysed before turning to the SPI- 24 for the remaining part of the paper.

\subsection{Spatial extent and trend of meteorological dry and wet spells}

In the latest years several European regions experienced wet events on meteorologically relevant time scale of seasons so 

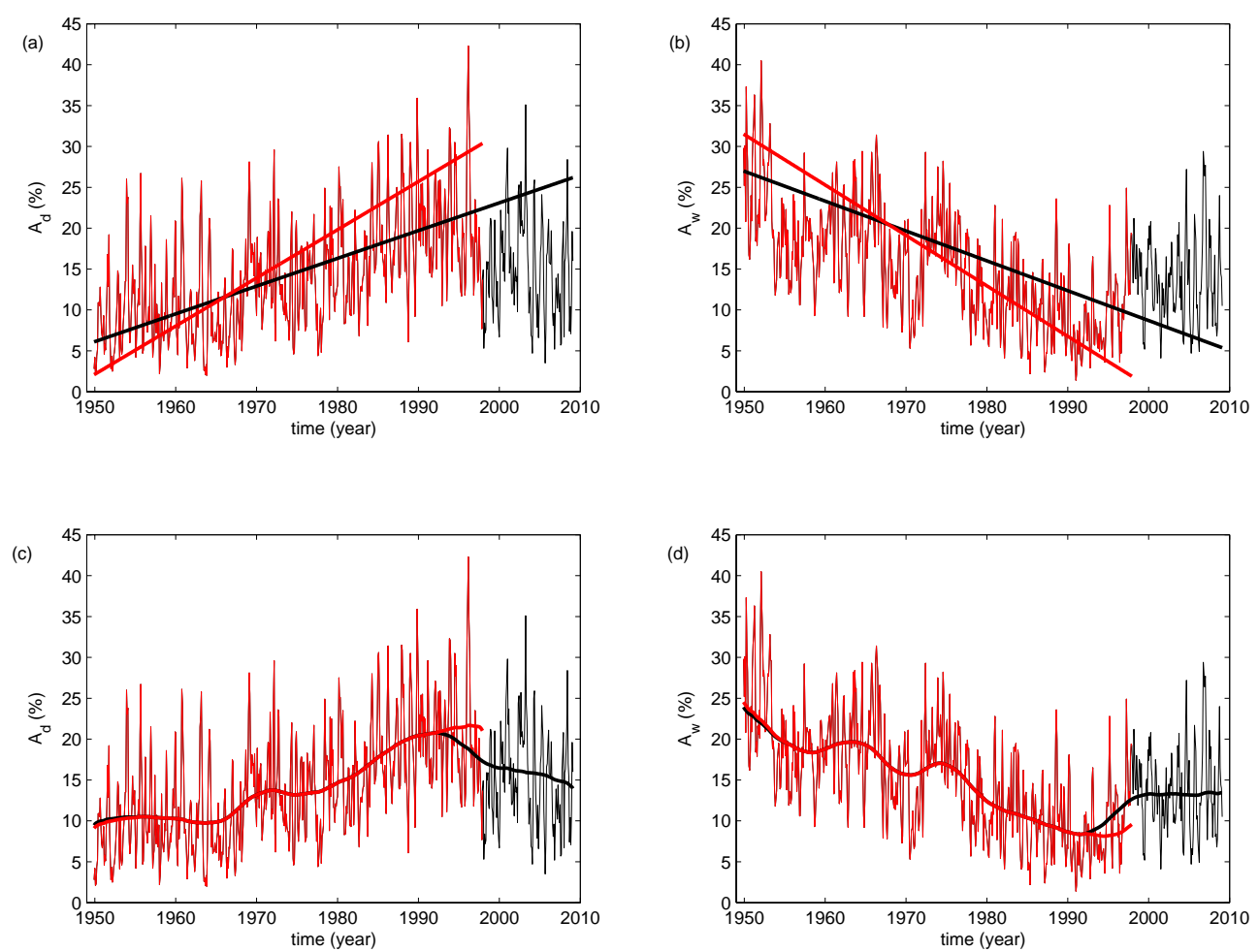

Fig. 1. Time behaviour of $A_{d}$ and $A_{w}$ from December 1949 to February 2009 for SPI-3 for (a) dryness and (b) wetness. The period January 1998- February 2009 is in black. Straight lines denote the fitting linear trends for the whole period December 1949-February 2009 (thick black line) and for the shorter period December 1949-December 1997 (thick read line). In (c) and (d) the same with the reconstructions of $A_{d}$ and $A_{w}$ using only the leading SSA principal component (thick black line for the whole period and thick red line for the shorter period).

that, at first, dryness and wetness analysis is based on SPI3. As that includes higher frequency fluctuations, we turn to SPI-24 in the following subsection to capture lower frequency and trend-like features; also, previous papers (Bordi and Sutera, 2001, 2004) are based on the hydrologically relevant drought and wetness analyses employing SPI-24.

Fractional cover and linear trend: dry and wet events in Europe are evaluated in terms of a gross measure of their area coverage by computing the percentage of grid points that are in dry and wet conditions, say $A_{d}$ and $A_{w}$, for each month of the time record. Let us consider drought occurrence identified by SPI-3 $<-1$ and wet events' occurrence by SPI-3 $>1$. Figure $1 \mathrm{a}, \mathrm{b}$ shows $A_{d}$ and $A_{w}$, respectively, as a function of time. Fitting linear trends for the periods December 1949-February 2009 (black straight lines) and December 1949-December 1997 (red straight lines) are displayed; values of the angular coefficients $\left(p_{1}\right)$ and intercepts $\left(p_{2}\right)$ with the corresponding statistics are listed in Table 1 . It can be seen that the area covered by dry events increased during the first 45 years of the time record, while in the latest decade or so there was a decrease. Furthermore, it appears that the area covered by wet events decreased in the first 45 years and increased during the latest decade. As a consequence, the positive linear trend (i.e. towards higher $A_{d}$ values) computed over the entire time section changes remarkably when the shorter time record December 1949-December 1997 is considered (Fig. 1a). A similar change is noticeable in Fig. 1b for the negative linear trend (i.e. towards lower $A_{w}$ values). Another interesting feature, which deserves attention, is that the overall long-term time evolutions of $A_{d}$ and $A_{w}$, due to the change occurred in the latest decade, are not well represented by linear functions. In fact, the percentages of the total variances explained by the fitting linear trends are greater, by about $15 \%$, for the shorter time period with respect to the full record length (see $R^{2}$ in Table 1).

Fractional cover and nonlinear trend: as the overall longterm time evolution of SPI- 3 area coverage, $A_{d}$ and $A_{w}$, during the latest decade is badly represented by linear functions, a nonlinear trend analysis needs to be introduced to extract the nonlinear behaviour of $A_{d}$ and $A_{w}$. Singular Spectrum Analysis (SSA) is applied to the time series using $M=70$ months. The reconstructions of $A_{d}$ and $A_{w}$ using only the leading principal component, $R_{1}(t)$, for the two dryness and wetness time sections are displayed in Fig. 1c, d. Note that that the nonlinear behaviour is mainly controlled by the multiyear periodicities characterizing $A_{d}$ and $A_{w}$ and, more importantly, by the change occurred in the latest decade. Notice that the percentages of the total variances explained by the reconstructed time series in all cases are higher than those obtained for the linear fittings (Tables 1 and 2). 
Table 1. Values of the angular coefficients and intercepts, with the corresponding error bands at $95 \%$ confidence level, of the linear trend detected in $A_{d}$ and $A_{w}$ time series for two time sections and for SPI-3 and SPI-24. The last two columns refer to the SSE and $R^{2}$ statistics.

\begin{tabular}{|c|c|c|c|c|c|}
\hline $\begin{array}{l}\text { Time scale and } \\
\text { time section }\end{array}$ & Variable & $\begin{array}{l}p_{1}\left(\text { year }^{-1}\right) \text { with } 95 \% \\
\text { confidence bounds }\end{array}$ & $\begin{array}{l}p_{2}\left(\text { year }^{-1}\right) \text { with } 95 \% \\
\text { confidence bounds }\end{array}$ & SSE & $R^{2}(\%)$ \\
\hline $\begin{array}{l}\text { 3-month } \\
\text { 1949-2009 }\end{array}$ & $A_{d}$ & $\begin{array}{l}0.1665 \\
(0.1399,0.1932)\end{array}$ & $\begin{array}{l}-315.3 \\
(-368.1,-262.6)\end{array}$ & $2.7 \times 10^{4}$ & 17.52 \\
\hline $\begin{array}{l}\text { 3-month } \\
\text { 1949-1997 }\end{array}$ & $A_{d}$ & $\begin{array}{l}0.2847 \\
(0.2509,0.3186)\end{array}$ & $\begin{array}{l}-547.9 \\
(-614.8,-481.1)\end{array}$ & $1.9 \times 10^{4}$ & 32.18 \\
\hline $\begin{array}{l}\text { 3-month } \\
\text { 1949-2009 }\end{array}$ & $A_{w}$ & $\begin{array}{l}-0.1949 \\
(-0.2200,-0.1699)\end{array}$ & $\begin{array}{l}400.8 \\
(351.2,450.4)\end{array}$ & $2.4 \times 10^{4}$ & 24.76 \\
\hline $\begin{array}{l}\text { 3-month } \\
\text { 1949-1997 }\end{array}$ & $A_{w}$ & $\begin{array}{l}-0.3209 \\
(-0.3527,-0.2891)\end{array}$ & $\begin{array}{l}648.6 \\
(585.9,711.4)\end{array}$ & $1.7 \times 10^{4}$ & 40.64 \\
\hline $\begin{array}{l}\text { 24-month } \\
\text { 1949-2009 }\end{array}$ & $A_{d}$ & $\begin{array}{l}0.3395 \\
(0.3091,0.3699)\end{array}$ & $\begin{array}{l}-655.9 \\
(-716.0,-595.8)\end{array}$ & $3.5 \times 10^{4}$ & 40.45 \\
\hline $\begin{array}{l}\text { 24-month } \\
\text { 1949-1997 }\end{array}$ & $A_{d}$ & $\begin{array}{l}0.5893 \\
(0.5579,0.6208)\end{array}$ & $\begin{array}{l}-1147.0 \\
(-1209.0,-1085.0)\end{array}$ & $1.6 \times 10^{4}$ & 70.25 \\
\hline $\begin{array}{l}\text { 24-month } \\
\text { 1949-2009 }\end{array}$ & $A_{w}$ & $\begin{array}{l}-0.3653 \\
(-0.3952,-0.3353)\end{array}$ & $\begin{array}{l}739.3 \\
(680.0,798.6)\end{array}$ & $3.4 \times 10^{4}$ & 44.72 \\
\hline $\begin{array}{l}\text { 24-month } \\
\text { 1949-1997 }\end{array}$ & $A_{w}$ & $\begin{array}{l}-0.6172 \\
(-0.6503,-0.584)\end{array}$ & $\begin{array}{l}1235.0 \\
(1169.0,1300.0)\end{array}$ & $1.8 \times 10^{4}$ & 69.88 \\
\hline
\end{tabular}

\subsection{Spatial extent and trend of hydrological drought and wetness}

SPI-24 is analysed to obtain the spatial distribution of hydrological drought and wetness and to analyse their trend. First, the fractional coverage of wet and dry regions and trend are determined and results are compared with SPI-3.

Fractional cover and linear trend: the dry and wet event area coverage in dry and wet conditions is identified by SPI$24<-1$ and SPI-24>1, respectively. Figure $2 a$, b shows the time evolution of percentage area covered by dryness and wetness, $A_{d}$ and $A_{w}$, with linear trends fitted for the periods December 1949-February 2009 (black straight lines) and December 1949-December 1997 (red straight lines). Table 1 lists angular coefficients $\left(p_{1}\right)$ and intercepts $\left(p_{2}\right)$ for comparison with SPI-3 results. The following results are noted: during the first 45 years the dry (wet) event area cover increased (decreased) dramatically, while the last decade shows a decrease (increase). Thus, the positive linear trend towards higher $A_{d}$ values for the whole period changes remarkably if only the shorter time record December 1949-1997 is considered (Fig. 2a). A similar change is noticeable in Fig. 2b for the negative linear trend (i.e. towards lower $A_{w}$ values).

Fractional cover and nonlinear trend: finally we note that, as for SPI-3, the overall long-term time evolution of SPI-24 area coverage during the latest decade is badly represented by linear functions. This nonlinear behaviour of $A_{d}$ and $A_{w}$ is captured by SSA ( $M=70$ month window); the reconstructions by leading SSA-principal component, $R_{1}(t)$, are shown in Fig. 2c, d. Note that the nonlinearity in the time series is
Table 2. SSE and $R^{2}$ statistics for the reconstructed $A_{d}$ and $A_{w}$ using only the first SSA principal component. Results are for the SPI time scales 3- and 24-months and the time sections 1949-2009 and 1949-1997.

\begin{tabular}{llll}
\hline $\begin{array}{l}\text { Time scale and } \\
\text { time section }\end{array}$ & Variable & SSE & $R^{2}(\%)$ \\
\hline $\begin{array}{l}\text { 3-month } \\
\text { 1949-2009 }\end{array}$ & $A_{d}$ & $2.3 \times 10^{4}$ & 31.49 \\
3-month & $A_{d}$ & $1.8 \times 10^{4}$ & 36.31 \\
1949-1997 & & & \\
3-month & $A_{w}$ & $1.8 \times 10^{4}$ & 44.72 \\
1949-2009 & & & \\
3-month & $A_{w}$ & $1.4 \times 10^{4}$ & 48.76 \\
1949-1997 & & & \\
24-month & $A_{d}$ & $1.2 \times 10^{4}$ & 79.35 \\
1949-2009 & & & \\
24-month & $A_{d}$ & $8.5 \times 10^{3}$ & 84.55 \\
1949-1997 & & & \\
24-month & $A_{w}$ & $9.6 \times 10^{3}$ & 84.51 \\
$\begin{array}{l}\text { 1949-2009 } \\
\text { 24-month }\end{array}$ & $A_{w}$ & $8.6 \times 10^{3}$ & 85.73 \\
1949-1997 & & & \\
\hline
\end{tabular}



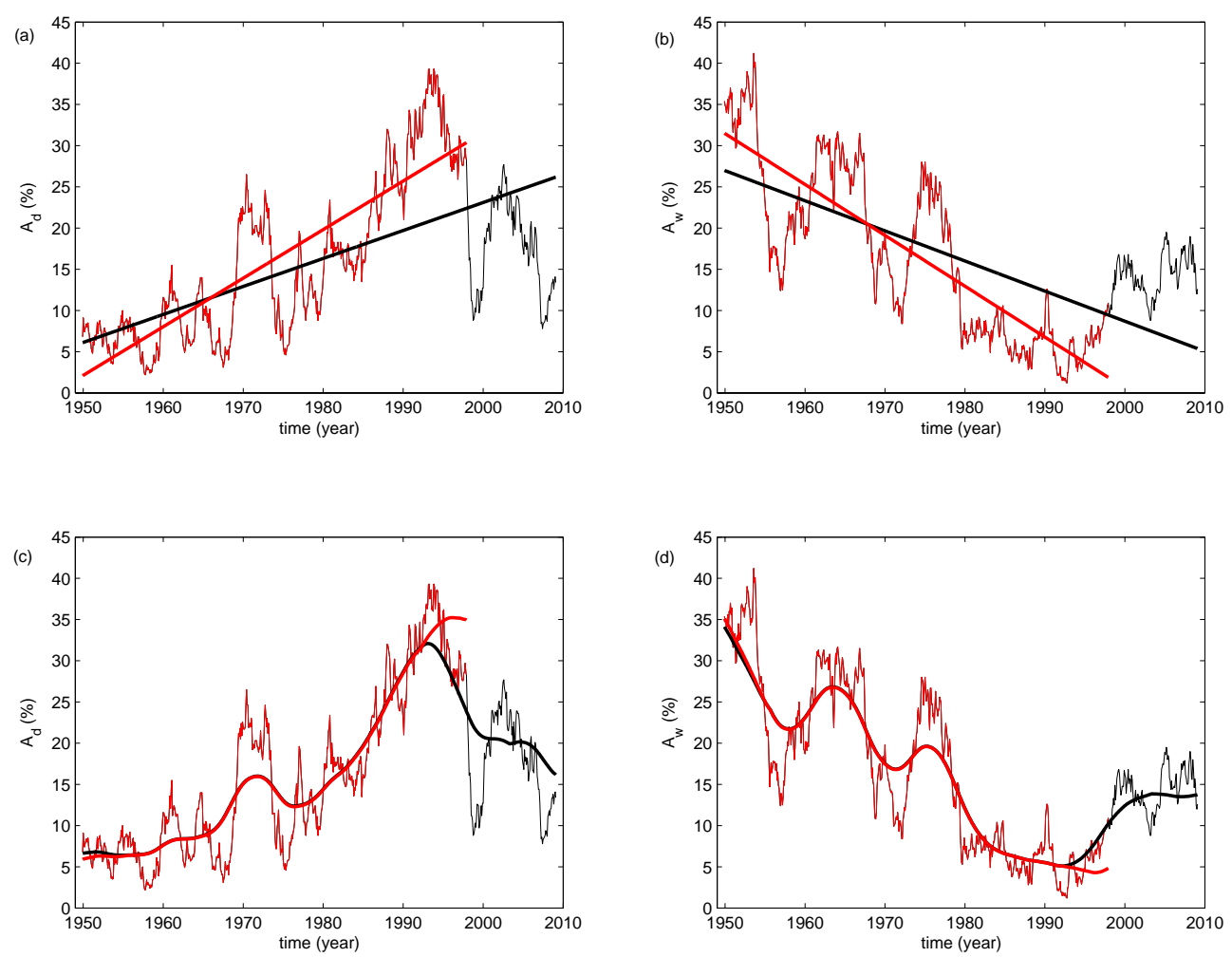

Fig. 2. As in Fig. 1 but for $A_{d}$ and $A_{w}$ computed using SPI-24.

dominated by multiyear periodicities and, more importantly, by the change that occurred in the latest decade; the percentages of total variances explained by the reconstructed time series are, in all cases, higher than the linear fits (Tables 1 and 2). Thus, results suggest that nonlinear fittings are more appropriate than the linear ones to detect long-term variability of dry and wet event area coverage.

\subsection{Space-time variability of linear and nonlinear trend in SPI time series}

The linear trend variability of the SPI-24 time series is analyzed for two different time sections: December 1949February 2009 and December 1949-December 1997. Principal component analysis of the SPI-24 field for spatial pattern and trend-distribution follows.

Linear trend variability in the SPI-24 time series: more details of the SPI-24 linear trend analysis are presented on grid point basis (Fig. 3) evaluating the angular coefficient of the linear fitting $p_{1}$ and the $R^{2}$-score or variance explained by the linear trend for the whole period 1949-2009 and for the shorter one (discarding the last decade). Negative values of $p_{1}$ denote a tendency towards drier periods, while positive ones towards wetter periods.

The following results are noted: (i) linear trend and variance explained are considerably weaker, if the last ten years is considered in the analysis; this is not surprising because of the trend reversal during the last decade. Only in a few regions the trend unveiled for the whole period is stronger when compared to the shorter period, i.e. Northern England, Central Spain or Eastern Turkey. (ii) The patterns of large trend and explained variance show similar spatial structure and do hardly change from the shorter to the whole time section.

Principal component analysis of the SPI-24 field: the preceding analysis is supplemented by the principal component analysis of the SPI-24 field to obtain the geographical distribution and time variability of drought and wetness.

The first loading (Loading 1) that explains $20.4 \%$ of the total variance, represents the leading spatial variability of drought and wetness, and the associated principal component score (PC-1) shows the time evolution (Fig. 4a, b). The loading pattern has high positive values in Central and Northern Europe, Balkans, Greece, Middle Orient, Northern Africa and Central Spain and it is almost identical to the first loading in Panel II shown in the paper by Bordi and Sutera (2001), where the authors provide the principal component analysis of the SPI-24 field in Europe for the period 1948-2000. The associated PC-1 (Fig. 4b) reveals a remarkable downward linear trend comparable to that shown by Bordi and Sutera (2001, their Panel III). It implies that the regions (mentioned above) with high positive loading values from the seventies onward have been affected by prevailing dry events. 

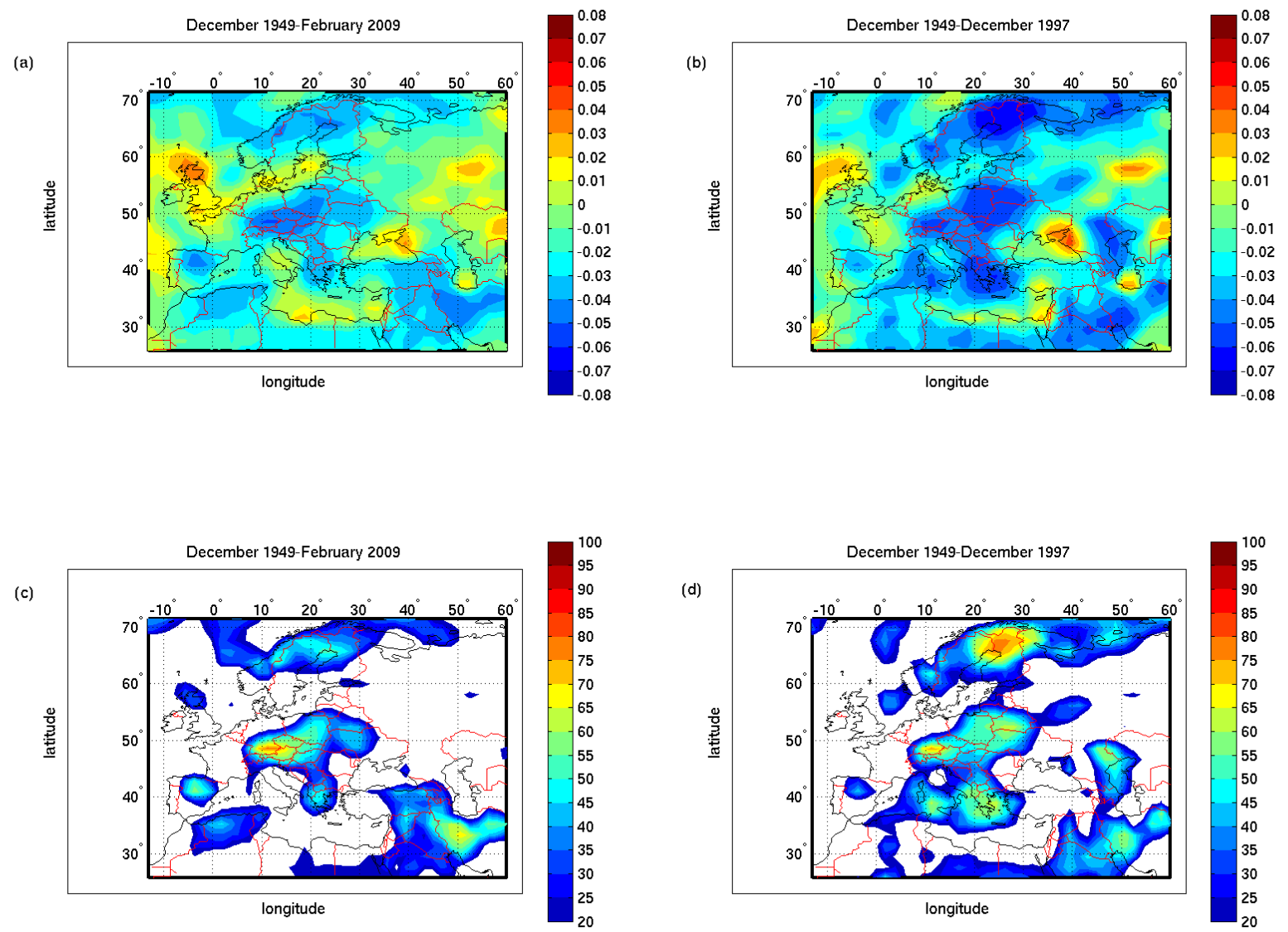

Fig. 3. Spatial distribution of the angular coefficient $p_{1}$ (in year $^{-1}$ ) of the linear trend fitting the SPI-24 time series for the two time sections: (a) December 1949-February 2009 and (b) December 1949- December 1997. Negative values of $p_{1}$ denote a tendency towards drier periods, while positive ones towards wetter periods. In (c) and (d) the corresponding $R^{2}$ statistics in \%; values of $R^{2}$ less than $20 \%$ are denoted in white.

However, it must be noted that during the latest decade the leading PC score shows a change, going up towards positive values (wetness in the above regions). Such a change provides a decrease of the slope of the linear fitting for the whole period compared to the shorter period (Fig. $4 \mathrm{~b}$ and Table 3). Moreover, when the whole time section is considered the $R^{2}$ is reduced of about $16 \%$ (from $83.41 \%$ to $67.01 \%$ ), suggesting that the linear fit becomes less suitable in explaining the variation of the data when the latest decade is taken into account. For this reason we have applied the SSA to the PC-1 time series setting $M=70$ months as before. The resulting reconstructed signal based only the leading SSA component, $R_{1}(t)$, is shown in Fig. $4 \mathrm{c}$ for both the whole and short period. Values of the $R^{2}$ in the two cases are similar and of about $92 \%$, suggesting a noticeable improvement of the fittings with respect to the linear ones. Moreover, it can be noted that the nonlinear behaviour of $R_{1}(t)$ is able to capture the dominant multi-year periodicities and, more importantly, the change occurred at the end of the nineties.

Thus, does the linear trend (detected for the short period) describe only the descending part of a periodic behaviour, which we do not sample because the shortness of the time series? A clear answer to this question, first raised by Bordi and Sutera (2001), is not possible at this stage of the analysis due to the limited size of the time record available. However, we can argue that the latest decade introduced a change in the time series of drought/wetness variability providing a hint of a long-term periodic behaviour.

Another question is related to the spatial variability of dryness/wetness: is the time behaviour of the SPI series well represented by PC-1 in some places (regions with high positive values in Loading 1) whereas it is not in other places (areas with low positive or negative values in Loading 1). To better illustrate this spatial variability we show, in the next section, the SPI time series at selected grid points and evaluate both linear and nonlinear long-term trends for comparison.

\section{Trend variability at selected grid points}

The trend of the first principal component score (Fig. 4b) is compared with single grid point trends (see Fig. 5) in 


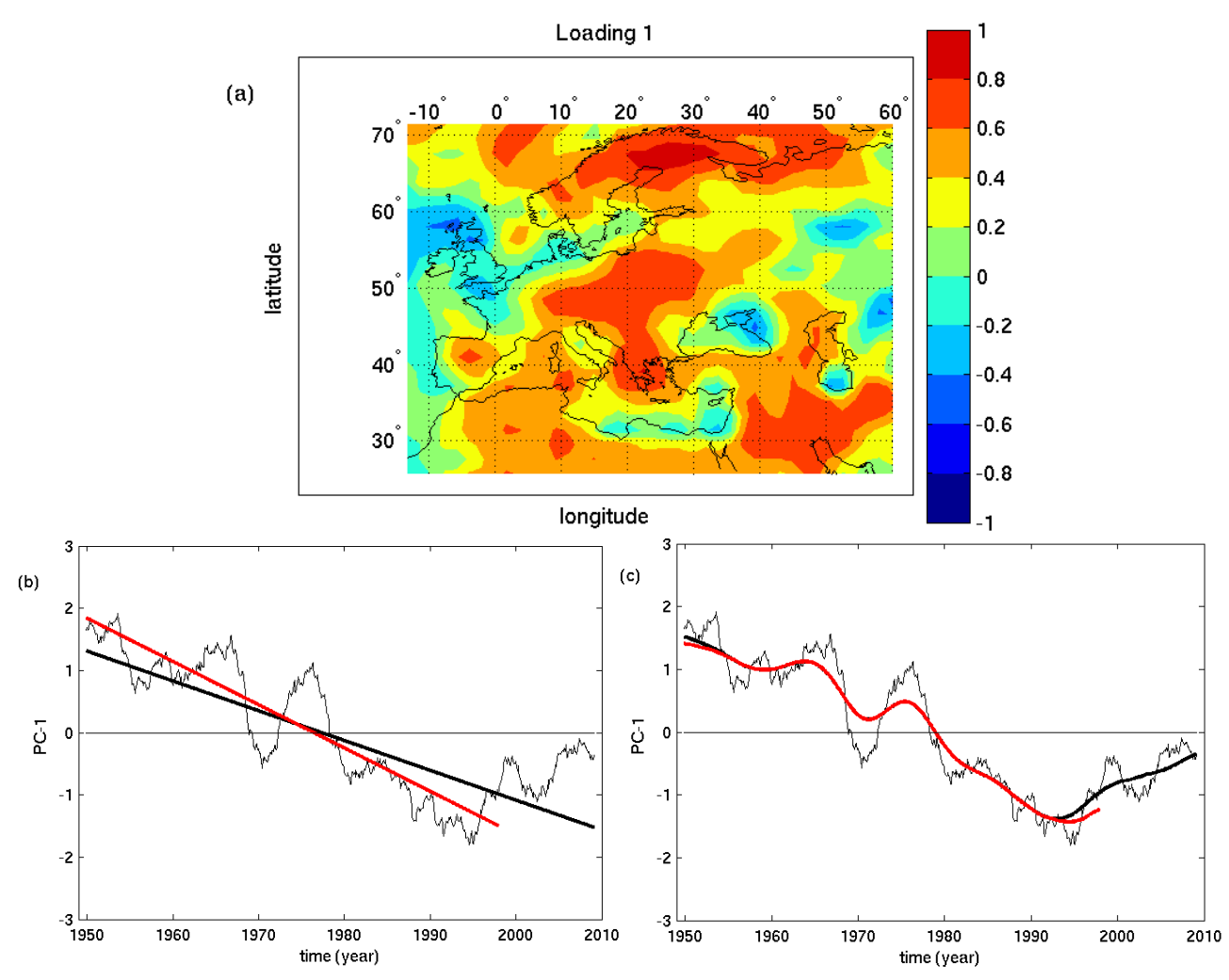

Fig. 4. Principal component analysis of SPI-24 field: (a) first loading, i.e. the first spatial pattern properly normalized that represents the correlations between the corresponding PC score and the SPI time series, and (b) first principal component score time series (PC-1). In (b) straight lines denote the fitting linear trends for the whole period (thick black line) and the shorter period (thick read line), while in (c) thick nonlinear lines are the reconstructed PC-1 time series using only the leading SSA component (as before black line for the whole period, read line for the shorter one).

Table 3. Values of the angular coefficients and intercepts, with the corresponding error bands at $95 \%$ confidence level, of the linear trend detected in the first PC score of the SPI-24 field for two time sections. The last two columns refer to the SSE and $R^{2}$ statistics.

\begin{tabular}{|c|c|c|c|c|c|}
\hline $\begin{array}{l}\text { Time scale and } \\
\text { time section }\end{array}$ & Variable & $\begin{array}{l}p_{1}\left(\text { year }^{-1}\right) \text { with } 95 \% \\
\text { confidence bounds }\end{array}$ & $\begin{array}{l}p_{2}\left(\text { year }^{-1}\right) \text { with } 95 \% \\
\text { confidence bounds }\end{array}$ & SSE & $R^{2}(\%)$ \\
\hline $\begin{array}{l}\text { 24-month } \\
\text { 1949-2009 }\end{array}$ & PC-1 & $\begin{array}{l}-0.0479 \\
(-0.0503,-0.0454)\end{array}$ & $\begin{array}{l}94.67 \\
(89.77,99.57)\end{array}$ & 234.2 & 67.01 \\
\hline $\begin{array}{l}\text { 24-month } \\
\text { 1949-1997 }\end{array}$ & PC-1 & $\begin{array}{l}-0.0695 \\
(-0.0721,-0.0670)\end{array}$ & $\begin{array}{l}137.4 \\
(132.3,142.4)\end{array}$ & 106.8 & 83.41 \\
\hline
\end{tabular}

Southern and Northern Europe (Greece 39.1 N, 22.5 E; Scandinavia 67.6 N, 26.3 E), and in Western and Eastern Europe (Northern England 58.1 N, 5.6 W; Northern Germany 52.4 N, $5.6 \mathrm{E})$. There is generally good agreement with the Loading 1 pattern and the PC-1 time evolution: (i) Greece and Scandinavian grid points closely follow the PC-1 time evolution (correlation 0.81 and 0.85 ) with trend reversal in the last decade. (ii) Northern Germany shows almost no change as it is located near the node of the Loading 1 pattern (the correlation coefficient with PC-1 is -0.05 ), and (iii) Northern England demonstrates the opposing trend in the initial decades (the correlation coefficient with PC-1 is -0.43 ). Note the different slopes of the linear trends for the selected grid points and time sections are those shown in Fig. 3: when the whole time record is considered a decrease of the negative slope, $p_{1}$, is observed in Greece and Scandinavia, a small change around zero characterizes Northern Germany, while an increase of the positive slope occurs in Northern England.

The reconstructed SPI signals with the leading SSA component at each grid points provide the nonlinear behaviours shown in Fig. 5 (thick black lines). The SSA technique captures the periodicities greater than 6 years and the long-term 

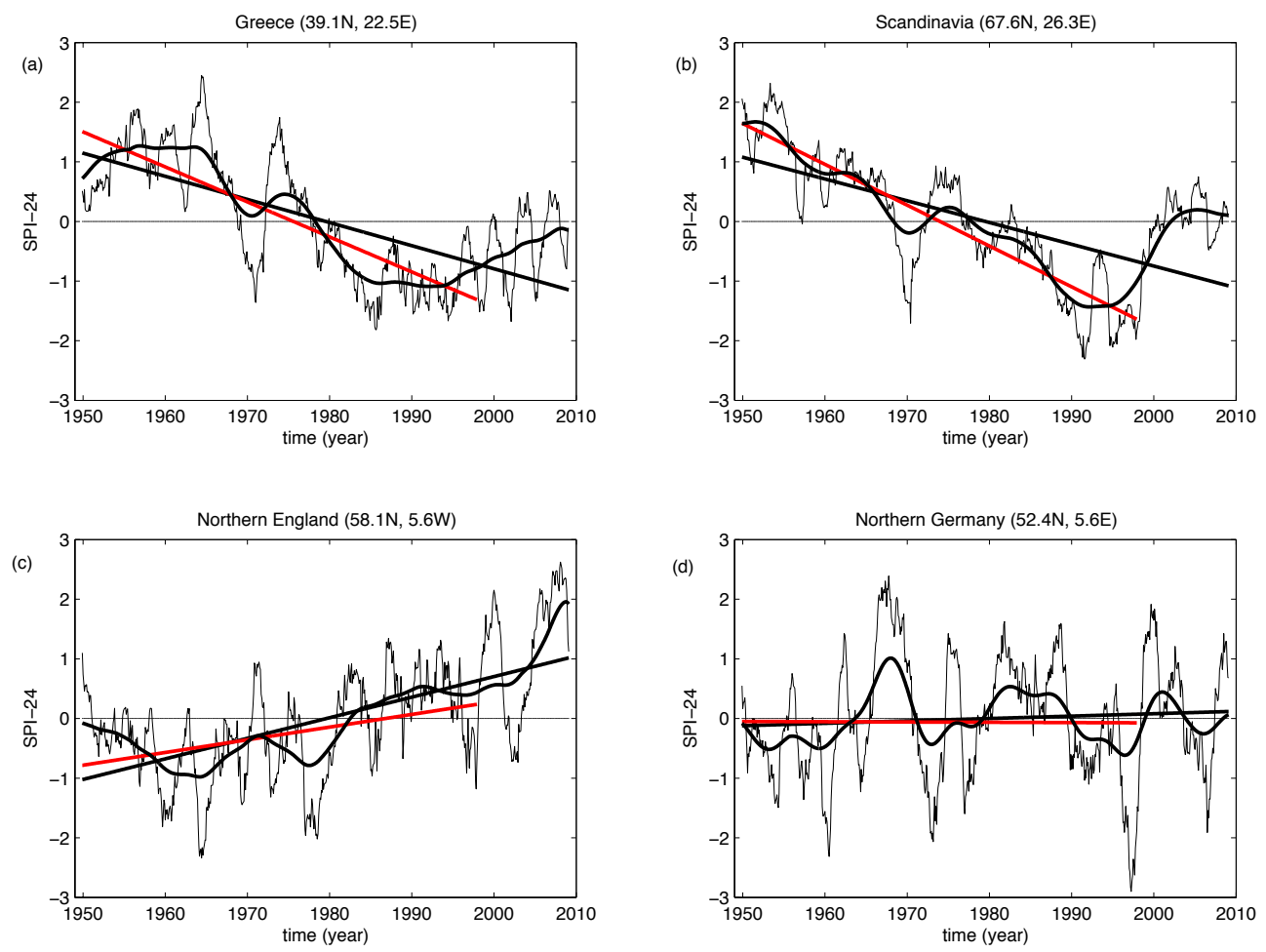

Fig. 5. SPI-24 time series for selected grid-points: (a) Greece, (b) Scandinavia, (c) Northern England, (d) Northern Germany. Straight lines are the fitting linear trends, while nonlinear thick lines the reconstructed SPI signals (whole period) using only the leading SSA component.

behaviour: in Greece and Scandinavia $R_{1}(t)$ series resemble the one obtained for PC-1 (Fig. 4c), while for the other two grid points they greatly depart from that.

Thus, results can be summarized as follows. Most of the SPI-24 time series in Eurasia during the last sixty years are characterized by linear trends towards negative values that strongly depend on the time section considered. In particular, it appears that changes in the slopes of the linear fittings are mainly controlled by the latest decade of data. However, these features also have a high spatial variability so that there are regions with no statistically significant linear trend, like Northern Germany, or others where the linear trend is towards positive values, like Northern England. For these reasons it is more suitable to consider a nonlinear fitting of the SPI time series that better represent the time variability of data, especially in recent years.

In the international literature there are several papers addressing the trend detection in drought episodes both at regional and large-scale level in Europe, using different data sources and methodologies (see for example Hisdal et al., 2001; Lloyd-Hughes and Saunders, 2002; Moreira et al., 2006; Briffa et al., 2009; Trnka et al., 2009), but no comprehensive study has been carried out with updated data. In agreement with our findings, all these efforts demonstrate the spatial variability of the detected trends and the crucial influence of the time records selected for the analysis. However, to increase the confidence on the reanalysis data here used and corroborate the obtained results, a careful comparison with observations should be done, even for case areas. This kind of additional analysis is not provided here for the lack of updated observations, but it is highly recommended and will be the topic of future investigations. For this purpose, it is important to stress the need for high quality data collection, quality control and updating of databases, including a broad spatial coverage.

\section{Conclusions and discussion}

An analysis of the linear and nonlinear trend in drought and wetness is provided for Europe using monthly precipitation data from NCEP/NCAR reanalysis data set. Meteorological and hydrological conditions are assessed applying the SPI on 3 and 24-month time scale, respectively. Two time sections are compared in the analysis: one from 1949 to 1997 and the other one includes the update of the last decade (to February 2009). First, the time series of drought and wetness area coverage for meteorological and hydrological conditions are analysed. Results show that the area covered by dry events (SPI-3 and SPI-24<-1) increased during the first 45 years of the time record, while there was a decrease in the latest ten years or so. On the contrary, the area covered by wet events (SPI-3 and SPI-24>1) shows the opposite behaviour. As a consequence, the linear trends, which characterize dry 
and wet area coverage $A_{d}$ and $A_{w}$, change remarkably, if the latest decade is taken into account or not. Thus, due to the change, which occurred in recent years, it appears more appropriate to fit $A_{d}$ and $A_{w}$ with nonlinear functions. For this purpose we apply singular spectrum analysis (SSA) to the area coverage time series and we reconstructed the signals using only the first SSA principal component. This approach allowed to better represent the variability of data, especially in recent years.

In the remaining part of the paper we focused the analysis on the space-time variability of hydrological dryness and wetness. The spatial variability of the slope of the linear functions fitting the SPI-24 time series is analysed, suggesting that most of the European regions are characterized by a negative trend towards drier conditions commencing in the seventies. However, the unveiled linear trends are highly dependent on the time section considered, in particular if the latest decade is taken into account or not. These features are better illustrated applying the PCA to the SPI-24 field and analysing the principal mode of variability, that is the first loading and PC score time series. The spatial structure of Loading 1 is very similar to that shown by Bordi and Sutera (2001, their Panel II) based on the period January 1950-December 2000. It should be interesting to verify the stability of such a pattern with respect to climate change applying the EOF analysis to the output of a general circulation model as discussed in Hansen and Sutera (1992). The PC-1 time series shows a change during the latest decade towards wet conditions; this is where the Loading 1 has high positive values and vice versa elsewhere. Thus, it appears more suitable to represent the long-term variability of data through a nonlinear fitting instead of the linear one. The application of the SSA to the PC-1 time series, reconstructing the signal using only the first SSA component, shows a noticeable improvement providing a hint of a long-term periodic behaviour. Nevertheless, further analyses are needed both to support the reliability of the NCEP/NCAR data (especially the confidence on reanalysis data against observations in the recent years and the possible impact of the assimilation system on trend detection) and to understand the physical causes leading to the observed change in precipitation, which is the basic variable of the SPI. About the last question, it should be of interest to investigate if an increase of the baroclinic activity at midlatitudes occurred in recent years leading to a change of the tropopause height (Bordi et al., 2002, 2004a) and of precipitation occurrence.

As a final analysis, we consider selected grid points for comparing the linear and nonlinear approach at different locations. Results highlight the differences between regions characterized by negative, positive or no linear trend. Uncertainty on the wave-like behaviour of the SPI-24 series in most of the European regions remains, since more data are necessary to corroborate this argumentation. This suggests how difficult it is to capture and quantify the long-term tendency in hydrological drought due to the high spatial variability and due to the dependence on the time section considered. These results should be taken into account in drought risk assessment and in planning proactive measures to limit the negative impacts of drought and wetness in Europe, also in relation to a changing climate.

The nonlinear trend analysis proposed here is able to capture the overall tendency of the SPI time series, and only partially it may provide an added value to drought forecasting. We like, in fact, to distinguish between "tendency" and "forecasting", which are different concepts both useful for planning proactive measures against the negative effects of droughts. For example, let us consider the grid point over Greece (Fig. 5a). During the latest decade the data suggest that there is a tendency towards near normal conditions, while the SPI index shows the occurrences of alternating near normal and moderate/severe droughts. Thus, the nonlinear component of the SPI series appears as a more appropriate measure than the linear fitting in capturing the overall tendency of the signal, though it is unable to properly forecast dry events.

On the other hand, forecasting drought remains a difficult task for the random character of the SPI signals that are characterized by multi-scale fluctuations. In previous works (Bordi et al., 2004b; Bordi and Sutera, 2007) we have investigated the potential predictability of drought events, assessed through the SPI, using Auto Regressive models, the Gamma Highest Probability (GAHP) method or the summation of periodic components that greatly contribute to the power spectrum variance of the SPI signals. The latest two methods provided interesting results; however, some limitations must be mentioned. In the case of GAHP method there is the assumption that precipitation for the future month is the most probable value described by the probability density function of precipitation for that month, while in the latest method needs several periodic components to properly reconstruct the SPI signal and extrapolate it forward.

Another approach that might be useful for planning proactive plans is the use of Hurst-Kolmogorov (HK) stochastic dynamics (Hurst, 1975; Montanari et al., 1997; Koutsoyiannis, 2003, 2006; Cohn and Lins, 2005; Fraedrich and Blender, 2003; Blender and Fraedrich, 2006, and references therein). Results here presented, in fact, are consistent with HK dynamics since the SPI time series appear to be not stationary and characterized by multi-scale fluctuations. Furthermore, the estimation of the Hurst coefficients of the SPI series for sample grid points (here not shown) provide values greater than 0.5 suggesting long-range persistence in the time series. However, such estimations appear to be unstable with respect to the method used for their computation and longer time records are requested to compute them with the needed accuracy. 
Acknowledgements. Data have been provided by the NOAACIRES Climate Diagnostic Center, Boulder, Colorado, from their web site at http://www.cdc.noaa.gov. We like to thank D. Koutsoyiannis, A. Montanari and two anonymous Reviewers for their fruitful comments and suggestions that contributed to improve the presentation of the paper.

Edited by: A. Montanari

\section{References}

Blender, R. and Fraedrich, K.: Long term memory of the hydrological cycle and river runoffs in China in a high resolution climate model, Int. J. Climatol., 26, 1547-1565, 2006.

Bordi, I. and Sutera, A.: Fifty years of precipitation: some spatially remote teleconnnections, Water Resour. Manag., 15, 247-280, 2001.

Bordi, I. and Sutera, A.: An analysis of drought in Italy in the last fifty years, Il Nuovo Cimento, 25C, 185-206, 2002.

Bordi, I., Dell'Aquila, A., Speranza, A., and Sutera, A.: Formula for a baroclinic adjustment theory of climate, Tellus, 54A, 260272, 2002.

Bordi, I. and Sutera, A.: Drought variability and its climatic implications, Global and Planet. Change, 40, 115-127, 2004.

Bordi, I., Dell'Aquila, A., Speranza, A., and Sutera, A.: On the midlatitude tropopause height and the orographic-baroclinic adjustment theory, Tellus, 56A, 278-286, 2004a.

Bordi, I., Fraedrich, K., Gerstengarbe, F.-W., Werner, P. C., and Sutera, A.: Potential predictability of dry and wet periods: Sicily and Elbe-Basin (Germany), Theor. Appl. Climatol., 77, 125-138, 2004b.

Bordi, I., Fraedrich, K., Petitta, M., and Sutera, A.: Large-scale assessment of drought variability based on NCEP/NCAR and ERA-40 re-analyses, Water Resour. Manag., 20, 899-915, 2006.

Bordi, I. and Sutera, A.: Drought monitoring and forecasting at large scale, in: Methods and Tools for Drought Analysis and Management, Series Water Science and Technology Library, edited by: Rossi, G., Vega, T., and Bonaccorso, B., Springer Verlag, vol. 62, 490 pp., 2007.

Briffa, K. R., van der Schrier, G., and Jones, P. D.: Wet and dry summers in Europe since 1750: evidence of increasing drought, Int. J. Climatol., doi:10.1002/joc.1836, 2009.

Broomhead, D. S. and King, G. P.: Extracting qualitative dynamics from experimental data, Physica D, 20, 217-236, 1986.

Cohn, T. A. and Lins, H. F.: Nature's style: naturally trendy, Geophys. Res. Lett., 32, L23402, doi:10.1029/2005GL024476, 2005.

Fraedrich, K.: Estimating the dimensions of weather and climate attractors, J. Atmos. Sci., 43, 419-432, 1986.

Fraedrich, K. and Blender, R.: Scaling of atmosphere and ocean temperature correlations in observations and climate models, Phys. Rev. Lett., 90, 1-4, 108501, 2003.

Ghil, M., Allen, M. R., Dettinger, M. D., et al.: Advanced spectral methods for climatic time series, Rev. Geophys., 40, 1.1-1.41, 2002.

Guttman, N. B.: Accepting the Standardised Precipitation Index: a calculation algorithm, J. Am. Water Resour. As., 35, 311-322, 1999.

Hansen, A. R. and Sutera, A.: Structure in the phase-space of a general circulation model deduced from empirical orthogonal func- tions, J. Atmos. Sci., 49, 320-326, 1992.

Hayes, M. J., Wilhelmi, O. V., and Knutson, C. L.: Reducing drought risk: bridging theory and practice, Natural Hazard Rev., 5, 106-113, 2004.

Heim Jr., R. R.: A Review of Twentieth-Century Drought Indices Used in the United States, B. Am. Meteorol. Soc., 83, 11491165, 2002.

Hisdal, H., Stahl, K., Tallasken, L. M., and Demuth, S.: Have streamflow droughts in Europe become more severe or frequent?, Int. J. Climatol., 21, 317-333, 2001.

Hurst, H. E.: Long term storage capacities of reservoirs, Trans. ASCE, 116, 776-808, 1951.

Janowiak, J. E., Gruber, A., Kondragunta, C. R., Livezey, R. E., and Huffman, G. J.: A comparison of the NCEP-NCAR reanalysis precipitation and the GPCP rain gauge-satellite combined dataset with observational error considerations, J. Climate, 11, 29602979, 1988.

Kalnay, E., Kanamitsu, M., Kistler, R., et al.: The NCEP-NCAR 40 year reanalysis project, B. Am. Meteorol. Soc., 77, 437-471, 1996.

Keyantash, J. and Dracup, J. A.: The quantification of drought: an evaluation of drought indices, B. Am. Meteorol. Soc., 83, 11671180, 2002.

Kistler, R., Kalnay, E., Collins, W., et al.: The NCEP-NCAR 50year reanalysis: monthly means CD-ROM and documentation, B. Am. Meteorol. Soc., 82, 247-267, 2001.

Koutsoyiannis, D.: Climate change, the Hurst phenomenon, and hydrological statistics, Hydrolog. Sci. J., 48(1), 3-24, 2003.

Koutsoyiannis, D.: Nonstationary versus scaling in hydrology, J. Hydrol., 324, 329-254, 2006.

Lloyd-Hughes, B. and Saunders, M. A.: A drought climatology for Europe, Int. J. Climatol., 22, 1571-1592, 2002.

McKee, T. B., Doesken, N. J., and Kleist J.: The relationship of drought frequency and duration to time scales, in: Proc. of the 8th Conference on Applied Climatology, 17-22 January, Anaheim, CA, American Meteorological Society, Boston, MA, 179-184, 1993.

Montanari, A., Rosso, R., and Taqqu, M.: Fractionally differenced ARIMA models applied to hydrologic time series: identification, estimation, and simulation, Water Resour. Res., 33, 1035-1044, 1997.

Moreira, E. E., Paulo, A. A., Pereira, L. S., and Mexia, J. T.: Analysis of SPI drought class transitions using loglinear models, J. Hydrol., 331, 349-359, 2006.

Pereira, L. S., Cordery, I., and Iacovides, I.: Coping with water scarsity, Addressing the challenges, Springer Netherlands, 2009.

Rencher, A. C.: Multivariate statistical inference and applications, John Wiley \& Sons, Inc., 1998.

Szinell, C. S., Bussay, A., and Szentimrey, T.: Drought tendencies in Hungary, Int. J. Climatol., 18, 1479-1491, 1998.

Trenberth, K. E. and Guillemot, C. J.: Evaluation of the atmospheric moisture and hydrological cycle in the NCEP/NCAR reanalyses, Clim. Dynam., 14, 213-231, 1998.

Trnka, M., Dubrovsky, M., Svoboda, M., Semerádová, D., Hayes, M., Zalud, Z., and Wilhite, D.: Developing a regional drought climatology for the Czech Republic, Int. J. Climatol., 29, 863883, 2009.

Vautard, R. and Ghil, M.: Singular spectrum analysis in nonlinear dynamics, with applications to paleoclimatic time series, Physica 
D, 35, 395-424, 1989.

Vicente-Serrano, S. M. and Cuadrat-Prats, J. M.: Trends in drought intensity and variability in the middle Ebro valley (NE of the Iberian peninsula) during the second half of the twentieth century, Theor. Appl. Climatol., 88, 247-258, 2007.
Wilhite, D. A., Hayes, M. J., Knutson, C., and Smith, K. H.: Planning for Drought: Moving from Crisis to Risk Management, J. Am. Water Resour. As., 36, 697-710, 2000.

Wilhite, D. A.: Combating Drought through Preparedness, Nat. Resour. Forum, 26, 275-285, 2002. 